\title{
Potential energy curves for the interaction of a low-energy positron with matter: The case $\mathrm{He}+\mathrm{e}^{+}$
}

\author{
José R. Mohallem ${ }^{\mathrm{a}, *}$, Flavia Rolim ${ }^{\mathrm{b}}$ \\ ${ }^{a}$ Laboratório de Átomos e Moléculas Especiais, Departamento de Física, ICEx, Universidade Federal de Minas Gerais, \\ P.O. Box 702, 30123-970 Belo Horizonte, MG, Brasil \\ ${ }^{\mathrm{b}}$ Departamento de Química, Universidade de Coimbra, FCT, 3004-535 Coimbra, Portugal
}

\begin{abstract}
In this introductory exploration of the title theme, we treat a positron as a light nucleus and work within the quasimolecule approximation to obtain, for the first time, adiabatic potential energy curves for its scattering by the He atom. We then show that different elastic and inelastic processes that contribute to the total scattering cross section can be rationalized in molecular terms as dissociation and non-adiabatic couplings. Particularly, some new insights on positronium yielding are presented.
\end{abstract}

(C) 2006 Elsevier Ltd. All rights reserved.

Keywords: Potential energy curves; Positron; Molecular model

\section{Introduction}

Studies of positron $\left(\mathrm{e}^{+}\right)$scattering by atoms and molecules are capable of generating substantial information on the target structure as well as on inelastic processes like positronium (Ps) formation, matter-antimatter interaction, etc. However, positron physical chemistry is still a theory under construction, being presently a collection of weakly connected results (Jean et al., 2003). The formation of bound complexes $\left[\mathrm{M} ; \mathrm{e}^{+}\right]$ (M being an atom or a molecule) is nowadays more likely to be predicted by calculations than detected in laboratory (Strasburger, 2004). On the other hand, scattering cross section experiments (for recent results see Marler et al., 2004) have gone far beyond theory (for recent results see Sueoka et al., 2000). Due to the special character of the complexes, the theoretical approaches for bound states (in general, all-body

\footnotetext{
*Corresponding author. Fax: + 553134995639.

E-mail address: rachid@fisica.ufmg.br (J.R. Mohallem).
}

calculations restricted to small systems) and for scattering show no clear connection. Furthermore, scattering calculations are commonly based on a coupled-state multi-channel framework in which the positron is taken as a third type particle, besides nuclei and electrons and the relevant channels corresponding to inelastic processes must be in the input instead of being an output of the theory.

In scattering theory of reaction chemistry, on the other hand, the approaches are quite easier because of the assumption that the electron distribution keeps stationary as the nuclei move, even for unbound states (the quasi-molecule model), yielding a potential energy surface (PES) or curve (PEC) for this motion. With the PESs for various states in hand, it becomes possible to treat the bound and scattering states in a unique approach. That is, scattering theory based on PESs is capable of performing the desired connection among theory of bound and unbound states and experiments. In fact, it has long been a challenge to theoreticians to generate PESs for positron motion (Schrader, 1992) that 
predict the different processes on an ab initio basis and permit cross sections estimation more easily.

We have found that treating the positron as a light nucleus (for an exploration of the same idea in another context see Karl et al., 1984) and performing an adiabatic separation of the electronic and nuclear motions, PESs for positron motion can be obtained that yield a lot of valuable information (Mohallem et al., 2004; Mohallem and Gonçalves, 2004) on the systems. Except for the large adiabatic correction to positron motion, everything else works as in standard molecular electronic structure calculations based on the BornOppenheimer approximation. In what follows, we consider a positron interacting with an $n$-electron, $m$ nuclei system $\mathrm{M}$ and refer to an electronic state of a complex $\left[\mathrm{M} ; \mathrm{e}^{+}\right]$irrespective of it being a scattering state or a bound one. In fact, the SEPs are determined by the electronic states involved, and can support bound states or not. In the unbound case, the complex will resemble the concept of quasi-molecule. We follow the approximation introduced in Mohallem et al. (2004), in which we consider separately the conservation of linear momentum in the atomic and positronic sub-systems. As a consequence, the non-relativistic Hamiltonian becomes, in conventional notation and atomic units (a.u., used throughout this work)

$H=\sum_{A}^{m} \sum_{i}^{n}\left(-\frac{\nabla_{i}^{2}}{2 \mathrm{M}_{A}} \delta_{A B}\right)-\frac{\nabla_{i}^{2}}{2}+V$,

where $V$ represents the Coulomb potential energy of all particles. The first term represents the nuclei and positron kinetic energy in terms of electronic operators. The $\delta_{A B}$ operator in practice forces the matrix elements of this term involving atomic orbitals $\varphi_{A}$ and $\varphi_{B}$ (centered on different nuclei) to vanish. This term will be strongly dominated by the positron kinetic energy, so that the contributions from other nuclei could in fact be neglected, as well as mass polarization contributions which are already neglected in the Hamiltonian (1). In consequence, the Hamiltonian becomes a pure electronic operator and the positron is treated on a common footing with the other nuclei except that a large mass correction is introduced for its motion. In the variational adiabatic approximation, the PESs for positron motion are obtained by the minimization of the quantity

$\varepsilon(R)=\frac{\langle\Phi|H| \Phi\rangle}{\langle\Phi \mid \Phi\rangle}$,

where $\Phi$ is a variational electronic wavefunction which depends parametrically on the set of nuclear (including positron) coordinates $R$, in the sense that $\Phi$ is normalizable for all $R$. In practice, the positron vector position could be seen as a set of three flat nuclear coordinates, in comparison with the rigid others. A reasonable procedure for future applications to molecules would be to consider the PES depending just on the positron coordinates and letting the other nuclear coordinates just to relax to account for each new positron position (Mohallem and Gonçalves, 2004). In the example below, this procedure is not necessary, however, since the positron interacts with a single $\mathrm{He}$ nucleus. Just one inter-nuclear coordinate $R$ will thus be used and the PESs will become PECs and referred to like this hereafter.

To solve Eq. (2), an electronic SCF-MO-CI (selfconsistent-field-molecular-orbital-configuration-interaction) method has been implemented as a simple modification of the Gamess code (Schimdt et al., 1993), referred to as the ISOTOPE program (Gonçalves and Mohallem, 2004). All the calculations reported here are performed with this code.

\section{Extracting information from the PECs}

The common belief that the positron complexes must always present a fully non-adiabatic behavior is, in fact, a wrong concept that comes from the Born-Oppenheimer culture. As we incorporate the adiabatic (but non-Born-Oppenheimer) effects to the theory, some surprisingly good results come out for common (Feagin and Briggs, 1986, 1988) or exotic (Mohallem et al., 2004; Mohallem and Gonçalves, 2004; Rost and Wintgen, 1992; Rolim et al., 2000) atoms and molecules. It has already been shown (Mohallem and Tostes, 2002; Mohallem, 2004) that using the approach described in the previous section is equivalent to an adiabatic approximation in which the total Hamiltonian is almost diagonal in the electronic states, so that just one of the two usual non-adiabatic coupling terms (O`Malley, 1971) survives. Particularly, the electronic ground state (g.s.) is usually found quite isolated from the other states. On the other hand, the question of choosing the proper reduced mass for the nuclear equation is not fully solved already (Rolim et al., 2004), so that this approximation is still less accurate for calculations of binding energies or other properties of the complexes in their bound state than totally non-adiabatic methods. However, the qualitative behavior of the electronic states is still an important source of knowledge for these systems and the approximate states obtained can be easily identified with those states coming from accurate methods. A first confirmation of this statement is that the cases of bound states predicted in the literature coincide with those PECs presenting minima that could support bound states in the present approach (see next section). Besides, in the same way as for typical molecules, correlation diagrams connecting separated atoms (SA) and united atoms asymptotic limits must preserve electronic symmetries and obey non-crossing rules. Finally, 
appearance of strong couplings will advance nonadiabatic behavior. In fact, almost all analogies with the Born-Oppenheimer theory of typical molecules can be explored (exceptions are semi-classical concepts like equilibrium geometry).

A determinant feature of the method is that the asymptotic behavior or threshold (SA, $R \rightarrow \infty$ ) of the low-lying PECs corresponds to exact or almost exact energies of the states of the dissociation products. Among these will be exact ground or excited states of Ps, since the approach accounts properly for the reduced mass effects (Mohallem et al., 2004), as well as very accurate states of the $\mathrm{M}$ atom or $\mathrm{M}^{+}$ion, allowed by electronic configuration-interaction calculations. Accordingly, some PECs show dissociation in an atom, in the ground or an excited state, plus the positron, while other PECs show dissociation in an atomic ion plus Ps, either excited or not. Mechanisms of atom excitation by a positron and of Ps formation are likely to be well described in this approach. An application to $\left[\mathrm{He} ; \mathrm{e}^{+}\right]$is made in the next section.

\section{The potential energy curves for $\left[\mathrm{He} ; \mathrm{e}^{+}\right]$}

The simplicity of the system considered here allows the prediction of the threshold of some low-lying PECs previously to calculations. We limit ourselves to $\mathrm{S}$ states, for simplicity. The energies of the $\mathrm{He}(1 \mathrm{~s}$, ns) Rydberg series vary in the range -2.903 a.u. [exact g.s. of $\mathrm{He}$ )] to -2.000 a.u. (exact g.s. of $\mathrm{He}^{+}$). Between these limits, just the two combinations

\section{(I) $\mathrm{He}(1 \mathrm{~s}, \mathrm{~ns})+\mathrm{e}^{+}$and (II) $\mathrm{He}^{+}(1 \mathrm{~s})+\mathrm{Ps}\left(\mathrm{n}^{\prime} \mathrm{s}\right)$}

are allowed, since the energies of the $\operatorname{Ps}\left(\mathrm{n}^{\prime} \mathrm{s}\right)$ states go from -0.250 a.u. to zero (both exact). The (I) curves will be related to the elastic positron scattering $(n=1$, g.s. $)$ and the collisional single excitations of the He atom. The (II) curves will correspond to ionization of the $\mathrm{He}$ atom along with positronium formation in its ground and excited states. Each singlet-triplet pair will have different threshold energies in case (I) but will be degenerated in case (II).

The PECs are obtained by running ISOTOPE on the single-double-configuration-interaction level. As justified above, an accurate quantitative behavior of the PECs for large inter-nuclear separations is fundamental, so we needed to expand the MOs with large basis sets of $\mathrm{s}$ and $\mathrm{p}$ type orbitals. For this we built generatorcoordinate basis sets (Mohallem, 1986; Mohallem et al., 1986) of 20s and 5p gaussian type orbitals (GTOs) for $\mathrm{He}$ and 20s and 1p GTOs for Ps (basis set details are available upon request). The energies of the dissociation products are calculated with the same method and basis sets. With these large basis sets, basis set superposition error is absent and we can identify the dissociation processes with combination of the energies of the products as well as by orbital occupation. PECs corresponding to some singlet (continuous) and a few triplet (dotted continuous) states appear in Fig. 1. The electronic g.s. (a) presents a minimum (not shown in the figure for reasons of scale), which is however not capable of supporting any bound state, according to the knowledge that the He atom cannot bind a positron in the g.s. (Schrader, 1979). Its asymptotic energy is the same as the $\mathrm{He}$ g.s., so that it corresponds to the dissociation scheme $\mathrm{He}(1 \mathrm{~s})+\mathrm{e}+$. On the other hand, though we have not yet calculated bound states in this introductory paper, it is easy to conclude by Landau and Lifshitz's (1965) rule that the first triplet PEC (g) is able to support at least one bound state. This is in accordance with the literature as well, including the prediction that in this case the two electrons will have parallel spins (Ryzhikh and Mitroy, 1998). There is also a singlet state (b) with the same asymptotic behavior, $\mathrm{He}^{+}(1 \mathrm{~s})+\mathrm{Ps}(1 \mathrm{~s})$ but which is dissociative, the same character of all other curves. In the threshold, the energy difference of the PECs $\mathbf{a}$ and $\mathbf{g}$ (or b) (see Table 1), (-2.2494+ $2.8978)=0.6484$ a.u., corresponds to the sum of the first ionization potential of $\mathrm{He}$ and the g.s. energy of Ps, $(0.8984-0.2500)=0.6484$ a.u. $=17,64 \mathrm{eV}$, which has long been known as the minimum energy that a positron needs to yield a Ps atom in a collision with an $\mathrm{He}$ atom (see for example Griffith, 1979). However, the $\mathbf{a} \rightarrow \mathbf{g}$ electronic excitation is forbidden by spin conservation so that the previous formation of a bound state will not be involved in Ps formation from the g.s. In the $\mathbf{a} \rightarrow \mathbf{b}$ process, the electron spin state is conserved and the state automatically dissociates with Ps formation.

Table 1 depicts the data for the PECs discussed above and some more states. At least for the low-lying states, the simple excitation pattern described above is kept so that the long-range behavior of the PECs appears like a tool for the analysis or the prediction of excitation processes of an atom or molecule by a slow positron.

Transitions between electronic states are more likely for higher states. A classical avoided crossing involving the singlet states $\mathbf{h}$ and $\mathbf{i}$ (see Fig. 1) occurs for $R \approx 3.7$ a.u. Apparently, due to this curve deformation, triplet $\mathbf{i}$ will converge to the same threshold energy of its singlet mate at much larger distances than those considered here. The interesting point is that while state h dissociates in $\mathrm{He}(1 \mathrm{~s}, 2 \mathrm{~s})+\mathrm{e}^{+}$, state $\mathbf{i}$ generates Ps through $\mathrm{He}^{+}(1 \mathrm{~s})+\operatorname{Ps}(2 \mathrm{~s})$. Similarly, curve $\mathbf{f}$ appears from a series of avoided crossings with higher states (apparently many more than those shown in the figure), some of them corresponding to Ps yielding as well. An immediate conclusion is that the cross section for Ps formation at energies higher than those of the lower three states will be strongly affected by non-adiabatic transitions. 


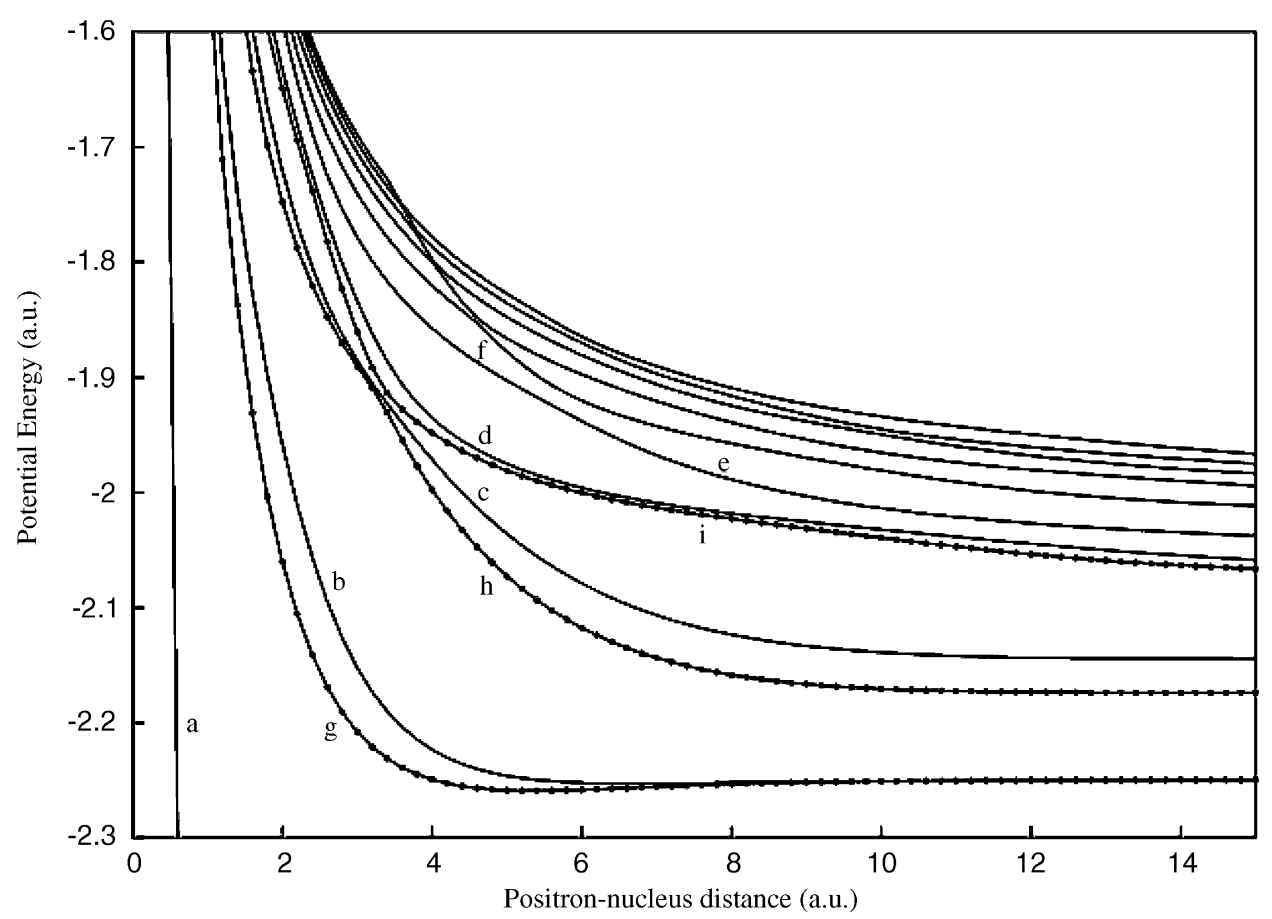

Fig. 1. Potential energy curves for some singlet (continuous) and triplet (dotted continuous) S states of [He; $\left.{ }^{+}\right]$. Further details are in the main text and in Table 1.

Table 1

Threshold energy and dissociation schemes for the PECs of Fig. 1

\begin{tabular}{lll}
\hline State & $\begin{array}{l}\text { PEC }(R \rightarrow \infty) \\
\text { a.u. }\end{array}$ & Dissociation \\
\hline${ }^{1}{ }^{1} \mathrm{~S}$ & -2.898 & $\mathrm{He}\left(1 \mathrm{~s}^{2}\right)+\mathrm{e}^{+}$ \\
$\mathrm{g}{ }^{3} \mathrm{~S}$ & -2.249 & $\mathrm{He}^{+}(1 \mathrm{~s})+\mathrm{Ps}(1 \mathrm{~s})$ \\
$\mathrm{b}{ }^{1} \mathrm{~S}$ & -2.249 & $\mathrm{He}^{+}(1 \mathrm{~s})+\mathrm{Ps}(1 \mathrm{~s})$ \\
$\mathrm{h}{ }^{3} \mathrm{~S}$ & -2.174 & $\mathrm{He}(1 \mathrm{~s}, 2 \mathrm{~s})+\mathrm{e}^{+}$ \\
$\mathrm{c}{ }^{1} \mathrm{~S}$ & -2.144 & $\mathrm{He}(1 \mathrm{~s}, 2 \mathrm{~s})+\mathrm{e}^{+}$ \\
${ }^{1} \mathrm{~S}$ & $-2.062^{\mathrm{a}}$ & $\mathrm{He}^{+}(1 \mathrm{~s})+\operatorname{Ps}(2 \mathrm{~s})$ \\
$\mathrm{d}{ }^{3} \mathrm{~S}$ & -2.062 & $\mathrm{He}^{+}(1 \mathrm{~s})+\operatorname{Ps}(2 \mathrm{~s})$ \\
$\mathrm{e}$ & -2.060 & $\mathrm{He}(1 \mathrm{~s}, 3 \mathrm{~s})+\mathrm{e}^{+}$ \\
\hline
\end{tabular}

${ }^{\mathrm{a}}$ Value estimated for very large $R$.

\section{Final remarks and prospects}

In abstract, we have generated, for the first time, PECs for positron motion in its interaction with an atom. The major advantage of such an approach is that some information that must enter as input in conventional calculations appears here as an output of the theory. A good example of this feature is the triplet character of the bound state reported by Ryzhikh and Mitroy (1998). An immediate consequence will be the calculation of bound states as vibrational states in the PECs that present minima. For this we will have to propose a proper reduced mass for the nuclear equation (Rolim et al., 2004). As a continuation of this work, we intend to perform applications to larger atoms and to molecules in order to generate complementary new data for the discussion of important issues not already fully understood like Ps yielding and pair annihilation in these systems (Gribakin, 2000).

\section{Acknowledgements}

We thank Marcus C.C. Simões for his help in the calculations and Dr. Maria Cristina A. Lopes for many useful discussions. We also acknowledge Brazilian agencies CNPq, Capes and Fapemig for financial support.

\section{References}

Feagin, J.M., Briggs, J.S., 1986. Molecular description of twoelectron atoms. Phys. Rev. Lett. 57, 984-987.

Feagin, J.M., Briggs, J.S., 1988. Molecular-orbital description of the states of two-electron systems. Phys. Rev. A 37, 4599-4613. 
Gonçalves, C.P., Mohallem, J.R., 2004. A new algorithm to handle finite nuclear mass effects in electronic calculations: the ISOTOPE program. J. Comput. Chem. 25, 1736-1739.

Gribakin, G.F., 2000. Mechanisms of positron annihilation on molecules. Phys. Rev. A 61 (article number 022720).

Griffith, T.C., 1979. Experimental aspects of positron collisions in gases. Adv. Atom. Mol. Phys. 15, 135-166.

Jean, Y.C., Mallon, P.E., Schrader, D.M., 2003. Principles and Applications of Positron and Positronium Chemistry. World Scientific, Singapore.

Karl, M.W., Nakanishi, H., Schrader, D.M., 1984. Chemical stability of positronic complexes with atoms and atomic ions. Phys. Rev. A 30, 1624-1628.

Landau, L.D., Lifshitz, M., 1965. Quantum Mechanics. Pergamon Press, Oxford (p. 167).

Marler, J.P., Barnes, L.D., Gilbert, S.J., Sullivan, J.P., Young, J.A., Surko, C.M., 2004. Experimental studies of the interaction of low energy positrons with atoms and molecules. Nucl. Instr. and Meth. B 221, 84-92.

Mohallem, J.R., 1986. A further study on the discretization of the Griffin-Hill-Wheeler equation. Z. Phys. D 3, 339-344.

Mohallem, J.R., 2004. Evidences of molecular structure beyond the Born-Oppenheimer approximation: the model Hamiltonian. J. Mol. Struc. (Theochem.) 709, 11-13.

Mohallem, J.R., Gonçalves, C.P., 2004. A note on geometry relaxation of molecular positron complexes. J. Chem. Phys. 121, 5553-5554.

Mohallem, J.R., Tostes, J.G.R., 2002. The adiabatic approximation to exotic leptonic molecules: further analysis and a nonlinear equation for conditional amplitudes. J. Mol. Struc. (Theochem.) 580, 27-32.

Mohallem, J.R., Dreizler, R.M., Trsic, M., 1986. A GriffinHill-Wheeler version of the Hartree-Fock equations. Int. J. Quantum. Chem. S 20, 45-55.
Mohallem, J.R., Rolim, F., Gonçalves, C.P., 2004. A molecular model for positron complexes: long-range effects on $2 \gamma$ pair-annihilation rates. J. Phys B: At. Mol. Opt. Phys. 37, $1045-1053$.

O 'Malley, T.F., 1971. Diabatic states of molecules - quasistationary electronic states. Adv. Atom. Mol. Phys. 7, 223-249.

Rolim, F., Braga, J.P., Mohallem, J.R., 2000. Unified description of chemical bonding in $\mathrm{H}_{2}$ isotopomers, including $\mathrm{Ps}_{2}$, $\mu_{2}$ and bi-excitons. Chem. Phys. Lett. 332, 139-144.

Rolim, F., Moreira, T., Mohallem, J.R., 2004. Heteroisotopic molecular behavior: the valence-bond theory of the positronium hydride. Braz. J. Phys. 34, 1197-1203.

Rost, J.M., Wintgen, D., 1992. Positronium negative ion: molecule or atom? Phys. Rev. Lett. 69, 2499-2502.

Ryzhikh, M., Mitroy, J., 1998. A metastable state of positronic helium. J. Phys. B: At. Mol. Opt. Phys. 31, 3465-3470.

Schimdt, M.W., Baldridge, K.K., Boatz, J.A., Elbert, S.T., Gordon, M.S., Jensen, J.H., Koseki, S., Matsunaga, N., Nguyen, K.A., Su, S.J., Windus, T.L., Dupuis, M., Montgomery, J.A., 1993. General atomic and molecular electronic structure system (Gamess). J. Comput. Chem. 14, 1347-1363.

Schrader, D.M., 1979. Semi-empirical polarization potential for low-energy positron-atom and positron atomic-ion interactions .1. Theory-hydrogen and the noble-gases. Phys. Rev. A 20, 918-932.

Schrader, D.M., 1992. Positronium hydride formation in collisions of positrons with molecular hydrogen. Theor. Chim. Acta 82, 425-434.

Strasburger, K., 2004. Positronic Formaldehyde - the configuration interaction study. Struct. Chem. 15, 415-420.

Sueoka, O., Kawada, M.K., Kimura, M., 2000. Total and positronium formation cross-sections in polyatomic molecules. Nucl. Instr. and Meth. B 171, 96-102. 